\title{
ON THE GEOMETRY OF THE RIEMANN TENSOR*
}

BY

\author{
R. V. CHURCHILL
}

\section{INTRODUCTION}

1. The problem. This paper deals with a problem which, for the Riemann tensor, or in general for a tensor of rank four with certain symmetry properties, is analogous to the well known problem which appears under many forms such as that of reducing a central conic to its principal axes or reducing a matrix to its classical canonical form. Here, as there, the problem may be stated as that of finding certain directions associated with the tensor, and determining the scalar quantities needed to complete the description of the tensor.

The space whose curvature tensor is considered here is a Riemannian space $V_{4}$ with a positive definite quadratic form.

A set of $n$ orthogonal directions in a $V_{n}$ was found by Ricci. $\dagger$ These are the principal directions of the first contracted Riemann tensor. $f$ Kretschmann§ has outlined a method which leads to a set of four directions, not in general orthogonal, and Struik $\|$ derived this set in a new way; but these directions have not been shown to be real when. the $V_{4}$ has a positive definite quadratic form.

A new procedure is adopted in this paper. The tensor is split up into two parts and the problem is solved for each part separately. The set of four orthogonal directions found for one of these parts coincides with those of Ricci (cf. \$18).

Six-vectors are used in the solution of the problem, so the theory of these vectors is reviewed and extended here.

2. Local coördinates. The vectors and tensors considered here are those at a given point of the $V_{4}$. Their components, denoted here by subscripts,

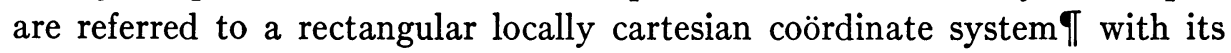
origin at the given point.

* Presented to the Society, December 1, 1928; received by the editors July 6, 1931; abstract published in the Bulletin of the American Mathematical Society, vol. 35 (1929), p. 154. This paper was prepared under the supervision of Professor G. Y. Rainich, University of Michigan.

$\dagger$ Ricci, Atti, Reale Istituto Veneto, vol. 63 (1904), p. 1233.

$\ddagger$ Eisenhart, Proceedings of the National Academy of Sciences, vol. 8 (1922), p. 24.

§ Kretschmann, Annalen der Physik, vol. 53 (1917), p. 592.

|| Struik, Journal of Mathematics and Physics, vol. 7 (1928), p. 193.

I For coördinate transformations which produce these local coördinates see Levi-Civita, Absolute Differential Calculus, Part II, \$11, or Eddington, Mathematical Theory of Relativity, 1924, §\$4, 36. 
Any term containing a Greek letter as a subscript is to be summed for the values $1,2,3,4$ of that subscript, unless another range of numbers is specified. Hence the scalar product of the vectors $x=\left(x_{1}, x_{2}, x_{3}, x_{4}\right)$ and $y=\left(y_{1}, y_{2}, y_{3}, y_{4}\right)$ can be written

$$
x y=x_{\alpha} y_{\alpha} .
$$

If $\lambda$ and $\mu$ are arbitrary numbers the family of vectors $\lambda x$ is called the direction of the vector $x$, and the family $\lambda x+\mu y$ is called the plane of $x$ and $y$. A vector $x$ is given in terms of the unit coördinate vectors $i=(1,0,0,0)$, $j=(0,1,0,0), k=(0,0,1,0), l=(0,0,0,1)$ by the equation

$$
x=x_{1} i+x_{2} j+x_{3} k+x_{4} l .
$$

Any new set of unit coördinate vectors can be obtained from $i, j, k, l$ by successive rotations in the coördinate planes: if the rotation

$$
i^{\prime}=i \cos \theta-j \sin \theta, j^{\prime}=i \sin \theta+j \cos \theta, \quad k^{\prime}=k, l^{\prime}=l
$$

in the $i, j$ plane through any angle $\theta$ is followed by an arbitrary rotation in the $i^{\prime}, k^{\prime}$ plane, and so on, the general rotation is obtained in six steps. By scalarly multiplying the members of (2.3) by $x$ and noting that $x_{1}=i x, x_{2}=j x$, etc., we obtain the relations between the old and new components of $x$ :

(2.4) $x_{1}^{\prime}=x_{1} \cos \theta-x_{2} \sin \theta, \quad x_{2}^{\prime}=x_{1} \sin \theta+x_{2} \cos \theta, x_{3}^{\prime}=x_{3}, x_{4}^{\prime}=x_{4}$.

3. The Riemann tensor. This definition of a tensor is used here: a tensor of rank $r$ is a scalar function of $r$ vectors which is linear in each of its vector arguments. ${ }^{*}$ Hence a tensor $R(x, y ; u, v)$ of rank four is a scalar function of its vector arguments $x, y, u, v$ which satisfies the linearity conditions

$$
\begin{aligned}
R(x+w, y ; u, v) & =R(x, y ; u, v)+R(w, y ; u, v), \cdots, \\
R(\lambda x, y ; u, v) & =\lambda R(x, y ; u, v), \cdots,
\end{aligned}
$$

where $\lambda$ is any scalar and $w$ is any vector, and where the dots indicate that these conditions apply to $y, u, v$ as well as to $x$.

Let $x, y, u$ and $v$ be written in the form (2.2); then when conditions (3.1) are applied to the tensor we find

$$
R(x, y ; u, v)=R_{\alpha \beta, \gamma \delta} x_{\alpha} y_{\beta} u_{\gamma} v_{\delta},
$$

where the numbers $R_{m, p q}(m, n, p, q=1,2,3,4)$ are the values assigned by the tensor to the unit coördinate vectors:

\footnotetext{
* The word linear here implies the properties (3.1). This definition of a tensor is given by Rainich, Two-dimensional tensor analysis without coördinates, American Journal of Mathematics, vol. 46 (1924), p. 77; also compare the definition given by Weyl, Raum, Zeit, Materie, 1923, §5.
} 


$$
\begin{aligned}
R_{11,11}=R(i, i ; i, i), \cdots & \\
R_{12,12} & =R(i, j ; i, j), \cdots, R_{12,34}=R(i, j ; k, l), \cdots .
\end{aligned}
$$

These numbers are the components of the tensor relative to our local coördinate system.

The Riemann tensor has the following fundamental properties:

$$
\begin{aligned}
& R(x, y ; u, v)=-R(y, x ; u, v)=-R(x, y ; v, u), \\
& R(x, y ; u, v)=R(u, v ; x, y), \\
& R(x, y ; u, v)+R(x, u ; v, y)+R(x, v ; y, u)=0 .
\end{aligned}
$$

By substituting the unit coördinate vectors for $x, y, u$, $v$, these properties become, in terms of components,

$$
\begin{aligned}
& R_{m n, p q}=-R_{n m, p q}=-R_{m n, q p}, \\
& R_{m n, p q}=R_{p q, m n}, \\
& R_{m n, p q}+R_{m p, q n}+R_{m q, n p}=0(m, n, p, q=1,2,3,4) .
\end{aligned}
$$

The first contracted Riemann tensor,

$$
R(x, u)=R(x, i ; u, i)+R(x, j ; u, j)+R(x, k ; u, k)+R(x, l ; u, l),
$$

is a tensor of rank two; its definition can also be written

$$
R_{m n}=R_{m \alpha, n \alpha} \quad(m, n=1,2,3,4) .
$$

The second contracted Riemann tensor is the number

$$
R=R_{\beta \alpha, \beta \alpha} .
$$

Our problem can now be reformulated as that of studying the geometry of a linear scalar function of four vectors in four-dimensional euclidean geometry, when this function satisfies (3.4), (3.5), (3.6).

\section{SiX-VECTORS}

4. Definitions and properties. The definitions and several of the properties of six-vectors given by Sommerfeld* are reviewed in this section. In addition to this we examine the uniqueness of a six-vector which is given by its components.

An elementary six-vector, called by Sommerfeld a special six-vector, is defined as a flat oriented area. It is determined by a plane, an area in this plane, and a direction of rotation about the origin in this plane, and these three characteristics are called the plane of the six-vector, its absolute value,

* Sommerfeld, Vierdimensionale Vektoralgebra, Annalen der Physik, vol. 32 (1910), p. 749. Also see Laue, Die Relativitätstheorie, 1921, p. 91. 
and its sense. We denote these vectors by small letters in the first part of the alphabet.

An elementary six-vector $c$ can be referred to our local coördinates by selecting any two four-vectors $x, y$ in the plane of $c$ such that the area of the parallelogram determined by $x$ and $y$ is equal to the absolute value of $c$. If $\phi$ is the least angle between $x$ and $y$ then just one of the vectors, say $x$, can be made to coincide with the other by a rotation through $\phi$ in the direction given by the sense of $c$. The components of this vector are used in the upper rows of the determinants

$$
\begin{aligned}
& \left|\begin{array}{ll}
x_{1} & x_{2} \\
y_{1} & y_{2}
\end{array}\right|=c_{12}, \quad\left|\begin{array}{ll}
x_{1} & x_{3} \\
y_{1} & y_{3}
\end{array}\right|=c_{13}, \quad\left|\begin{array}{ll}
x_{1} & x_{4} \\
y_{1} & y_{4}
\end{array}\right|=c_{14}, \\
& \left|\begin{array}{ll}
x_{2} & x_{3} \\
y_{2} & y_{3}
\end{array}\right|=c_{23}, \quad\left|\begin{array}{ll}
x_{4} & x_{2} \\
y_{4} & y_{2}
\end{array}\right|=c_{42}, \quad\left|\begin{array}{ll}
x_{3} & x_{4} \\
y_{3} & y_{4}
\end{array}\right|=c_{34},
\end{aligned}
$$

which are called the components of $c$. The invariant expression

$$
{c_{12}}^{2}+{c_{13}}^{2}+{c_{14}}^{2}+{c_{23}}^{2}+{c_{42}^{2}}^{2}+{c_{34}}^{2}=c^{2}
$$

is the square of the area of the parallelogram, or the square of $c$. From (4.1) it follows that the components of any elementary six-vector satisfy the relation

$$
c_{12} c_{34}+c_{13} c_{42}+c_{14} c_{23}=0 .
$$

Corresponding to $c$ there is an elementary six-vector $\bar{c}$ with the same absolute value whose plane is absolutely perpendicular* to the plane of $c$; the six-vector $-c$ also corresponds in this way to $c$. If the sense of $c$ is properly chosen, its components are related to those of $c$ in the following way: $\dagger$

$$
\text { (4.4) } \quad \bar{c}_{12}=c_{34}, \quad \bar{c}_{13}=c_{42}, \quad \bar{c}_{14}=c_{23}, \quad \bar{c}_{23}=c_{14}, \quad \bar{c}_{42}=c_{13}, \quad \bar{c}_{34}=c_{12} \text {. }
$$

The elementary six-vector $\bar{c}$ is called the dual of $c$; it follows from (4.4) that the dual of $\bar{c}$ is again $c$.

The general six-vector, denoted here by a capital letter in the first part of the alphabet, is determined by two elementary six-vectors whose planes are absolutely perpendicular to each other. $\neq$ Its components are the sums of the corresponding components of its elementary six-vectors. If $c$ is any unit elementary six-vector and $\lambda, \mu$ are any two numbers, then the components of any general six-vector $C$ are

* Two planes are absolutely perpendicular if each vector of one is perpendicular to every vector of the other.

$\dagger$ These relations are derived by Sommerfeld, loc. cit., p. 756.

$\ddagger$ This definition is slightly modified in the paragraph preceding Theorem 2 . 


$$
C_{12}=\lambda c_{12}+\mu \bar{c}_{12}, \quad C_{13}=\lambda c_{13}+\mu \bar{c}_{13}, \cdots, C_{34}=\lambda c_{34}+\mu \bar{c}_{34} .
$$

According to (4.1) and (4.4) these components can also be written

$$
C_{12}=\lambda\left|\begin{array}{ll}
x_{1} & x_{2} \\
y_{1} & y_{2}
\end{array}\right|+\mu\left|\begin{array}{ll}
x_{3} & x_{4} \\
y_{3} & y_{4}
\end{array}\right|, \quad C_{13}=\lambda\left|\begin{array}{ll}
x_{1} & x_{3} \\
y_{1} & y_{3}
\end{array}\right|+\mu\left|\begin{array}{ll}
x_{4} & x_{2} \\
y_{4} & y_{2}
\end{array}\right|, \cdots,
$$

where $x$ and $y$ are two four-vectors which determine the unit elementary sixvector $c$. The numbers $\lambda$ and $\mu$ are the absolute values of $\lambda c$ and $\mu c$; they are called the characteristic numbers of $C$.

The six-vector $\bar{C}$, whose elementary six-vectors are the duals of $\lambda c$ and $\mu \bar{c}$, is called the dual of $C$. Its components are

$$
\bar{C}_{12}=\lambda \bar{c}_{12}+\mu c_{12}, \quad \bar{C}_{13}=\lambda \bar{c}_{13}+\mu c_{13}, \cdots, \bar{C}_{34}=\lambda \bar{c}_{34}+\mu c_{34},
$$

and from (4.5) and (4.4) it follows that

$$
\bar{C}_{12}=C_{34}, \bar{C}_{13}=C_{42}, \bar{C}_{14}=C_{23}, \bar{C}_{23}=C_{14}, \bar{C}_{42}=C_{13}, \bar{C}_{34}=C_{12} \text {. }
$$

The law of transformation of the components of six-vectors follows from (4.6) and (2.4). After the rotation (2.3) the new components of $C$ are given in terms of the old by the equations

$$
\begin{array}{ll}
C_{12}^{\prime}=C_{12}, & C_{34}^{\prime}=C_{34}, \\
C_{13}^{\prime}=C_{13} \cos \theta-C_{23} \sin \theta, & C_{23}^{\prime}=C_{13} \sin \theta+C_{23} \cos \theta, \\
C_{14}^{\prime}=C_{14} \cos \theta-C_{24} \sin \theta, & C_{24}^{\prime}=C_{14} \sin \theta+C_{24} \cos \theta,
\end{array}
$$

where $C_{m n}=-C_{n m}$.

LEMMA 1. If any six numbers depend upon the coördinate system in such a way that they transform like the components of a six-vector then there is at least one six-vector with these numbers as components.

By four successive transformations of the type (4.9) it is always possible to determine the four angles involved so as to make all but the first and last of the six numbers $C_{12}, C_{13}, \cdots, C_{34}$ vanish. The new values $\left(C_{12}^{\prime}, 0,0,0,0, C_{34}^{\prime}\right)$ correspond to a new set $i^{\prime}, j^{\prime}, k^{\prime}, l^{\prime}$ of coördinate vectors, and two vectors can be selected in the $i^{\prime}, j^{\prime}$ plane and two in the $k^{\prime}, l^{\prime}$ plane so as to determine a six-vector whose components have these values. The components of this six-vector relative to the original coördinate system.are the original six numbers.

Before discussing the uniqueness of this six-vector let us introduce the scalar product of two six-vectors,

(4.10) $\quad B C=B_{12} C_{12}+B_{13} C_{13}+B_{14} C_{14}+B_{23} C_{23}+B_{42} C_{42}+B_{34} C_{34}$;

this expression in the components of $B$ and $C$ is invariant under transformations of the type (4.9). The relation (4.3) can now be written 


$$
c \bar{c}=0,
$$

and it follows from (4.5) and (4.7) that

$$
\begin{aligned}
C^{2} & =\lambda^{2}+\mu^{2}, \\
C \bar{C} & =2 \lambda \mu .
\end{aligned}
$$

According to the definition of a general six-vector, $C$ is elementary if $\lambda$ or $\mu$ is zero. It follows from (4.13) that $C$ is elementary if $C \bar{C}=0$, and, since the converse is given by (4.11), the following theorem is proved.

THEOREM 1. A necessary and sufficient condition that a six-vector be elementary is the vanishing of the product of this vector by its dual.

If the components of the six-vector in Lemma 1 satisfy (4.3) then when all except the first and last are made to vanish it will follow that $C_{12}^{\prime} C_{34}^{\prime}=0$. According to the definition of an elementary six-vector there is just one with components $\left(C_{12}^{\prime}, 0,0,0,0,0\right)$, so an elementary six-vector is uniquely determined by its components.

According to Lemma 1 there exists a unit elementary six-vector $c$ and two numbers $\lambda, \mu$ such that the six numbers $C_{m n}$ are the components of the sixvector determined by $\lambda c$ and $\mu c$ :

$$
C_{m n}=\lambda c_{m n}+\mu \bar{c}_{m n} .
$$

$C_{m n}$ determine the values of $C^{2}$ and $C \bar{C}$, and (4.12), (4.13) give the relations

$$
(\lambda+\mu)^{2}=C^{2}+C \bar{C},(\lambda-\mu)^{2}=C^{2}-C \bar{C} .
$$

Bearing in mind that an elementary six-vector is uniquely determined by its components it is easy to see that (4.15) and the six equations (4.14) determine $\lambda, \mu, c_{m n}$ in such a way that there is just one pair of elementary six-vectors $\lambda c, \mu \bar{c}$, provided $\lambda^{2} \neq \mu^{2}$.

If $\lambda^{2}=\mu^{2}$, there is a two-parameter family of unit elementary six-vectors any one of which, together with its dual and the number $\lambda$, determines a sixvector with the numbers $C_{m n}$ as components. If $b$ and $c$ are any two unit elementary six-vectors of this family, then

$$
C_{m n}=\lambda\left(b_{m n}+\bar{b}_{m n}\right)=\lambda\left(c_{m n}+\bar{c}_{m n}\right),
$$

when $\lambda=\mu$. We now modify our definition of a general six-vector to this extent: when $\lambda^{2}=\mu^{2}$, we call the six-vector determined by $b, \bar{b}, \lambda$ the same as that determined by $c, \bar{c}, \lambda$. The following theorem is then true.

ThEOREM 2. Six numbers which transform like the components of a sixvector are the components of a unique six-vector $C$; the components uniquely determine the elementary six-vector parts $\lambda c, \mu \bar{c}$ of $C$ except when $\lambda^{2}=\mu^{2}$. 
Since $A_{m n}+B_{m n}$ transform like the components of a six-vector when $A$ and $B$ are six-vectors, it now follows that these numbers are the components of a unique six-vector $C$, called the sum of $A$ and $B$ :

$$
C=A+B \text { if } C_{m n}=A_{m n}+B_{m n} .
$$

The distributive laws for duals and scalar products,

$$
\bar{C}=\bar{A}+\bar{B}, D C=D A+D B,
$$

follow at once. As a further consequence of (4.16), we may write (4.5) and (4.7) as

$$
\begin{aligned}
& C=\lambda c+\mu \bar{c}, \\
& \bar{C}=\lambda \bar{c}+\mu c .
\end{aligned}
$$

5. Further properties of six-vectors. If the elementary six-vectors $b$ and $c$ are given by two pairs of four-vectors $x, y$ and $u, v$ then their scalar product is given by

$$
b c=\left|\begin{array}{cc}
x u & x v \\
y u & y v
\end{array}\right|
$$

All quantities involved here are invariants, so any coördinate system can be used to prove this. Let coördinate vectors be chosen so that $i$ falls along $x$, and $j$ in the plane of $x$ and $y$; then $x=\left(x_{1}, 0,0,0\right)$ and $y=\left(y_{1}, y_{2}, 0,0\right)$ and the determinant reduces to $b_{12} c_{12}$ which is the product $b c$.

We shall call two six-vectors perpendicular if their scalar product vanishes.

THEOREM 3. The planes of two perpendicular elementary six-vectors are either conditionally or absolutely perpendicular.

To prove this we represent one of these six-vectors by two perpendicular four-vectors $x$ and $y$, and the other by $u$ and $v$, and then select coördinate vectors so that $x=\left(x_{1}, 0,0,0\right), y=\left(0, y_{2}, 0,0\right)$. Then according to $(5.1)$, the condition that the two six-vectors be perpendicular reduces to

$$
u_{1} v_{2}-v_{1} u_{2}=0
$$

and from this it follows that

$$
\left(u_{1} v_{2}-v_{1} u_{2}\right) y_{2}=\left(u_{1} v-v_{1} u\right) y=0 .
$$

The last equation, together with the identity

$$
\left(u_{1} v-v_{1} u\right) x=0,
$$

shows that, unless $u_{1}=v_{1}=0$, the vector $u_{1} v-v_{1} u$ in the plane of $v$ and $u$ is 
perpendicular to both $x$ and $y$, and hence to every vector in the plane of $x$ and $y$. If $u_{1}=v_{1}=0$, then $x$ is perpendicular to every vector in the plane of $u$ and $v$. Hence there is at least one vector in one plane perpendicular to all vectors of the other, so the two planes are perpendicular.

Let us now eliminate the double subscripts which have been used to denote the components of six-vectors. We number the six pairs of subscripts according to the table

$\begin{array}{lrrrrrr}\text { pair: } & 12 & 13 & 14 & 23 & 42 & 34 \\ \text { number: } & 1 & 2 & 3 & 4 & 5 & 6\end{array}$

and use the numbers as subscripts instead of the pairs. In what follows then $C_{1}, C_{2}, \cdots, C_{6}$ are written for $C_{12}, C_{13}, \cdots, C_{34}$, respectively.

In terms of this notation the properties (4.8), (4.10) become, respectively,

$$
\begin{aligned}
\bar{C}_{s} & =C_{7-8} & (s=1,2, \cdots, 6), \\
B C & =B_{1} C_{1}+\cdots+B_{6} C_{6}=B_{p} C_{p} & (\rho=1,2, \cdots, 6),
\end{aligned}
$$

and the condition that $c$ be elementary can be written

$$
c_{\rho} \bar{c}_{\rho}=c_{\rho} c_{7-p}=0 \quad(\rho=1,2, \cdots, 6) .
$$

It is evident that for $\rho=1,2, \cdots, 6$, the sum $B_{7_{-\rho}} C_{7_{-\rho}}$ is the same as the sum $B_{\rho} C_{\rho}$, so the relation

$$
\bar{B} \bar{C}=B C
$$

follows from (5.3) and (5.4). In the same way we find that

$$
B \bar{C}=\bar{B} C \text {. }
$$

The six elementary six-vectors $I_{1}, I_{2}, \cdots, I_{6}$ whose components relative to a given coördinate system are $(1,0,0,0,0,0),(0,1,0,0,0,0), \cdots,(0,0$, $0,0,0,1)$, are called the unit coördinate six-vectors. Each of these has a coördinate plane for its plane, e.g., the $i, j$ plane is the plane of $I_{1}$ and the $i, k$ plane is the plane of $I_{2}$. These unit coördinate six-vectors are mutually perpendicular; moreover, $I_{1}=I_{6}, I_{2}=I_{5}, I_{3}=I_{4}$. From the definition of the sum of six-vectors it follows that

$$
C=C_{\rho} I_{\rho} \quad(\rho=1,2, \cdots, 6) .
$$

A condition under which a set of six-vectors form a set of unit coördinate sixvectors is given by the following theorem.

THEOREM 4. If $a, b, c$ and their duals $\bar{a}, \bar{b}, \bar{c}$ are six mutually perpendicular unit six-vectors, the intersections of their planes determine a set of unit coördinate four-vectors for which $a, b, c, \bar{a}, \bar{b}, \bar{c}$ are the unit coördinate six-vectors. 
These vectors are elementary according to Theorem 1 . The plane of $\bar{a}$ is absolutely perpendicular to that of $a$. The condition $a c=0$ means that the plane of $c$ is perpendicular to that of $a$ (Theorem 3); it must be conditionally perpendicular since $c$ and $\bar{a}$ can not have a common plane because of the hypothesis $c \bar{a}=0$. Hence in the plane of $c$ there is a unit four-vector $l$ which is perpendicular to the plane of $a$; it follows that $i$ is common to the planes of $c$ and $\bar{a}$. From the condition $c \bar{a}=0$ we find in like manner that there is a unit vector $i$ common to the planes of $c$ and $a$. Similarly $\bar{c}$ and $\bar{a}$ determine $k$, and $\bar{c}$ and $a$ determine $j$. When $i, j, k, l$ are adjusted as to sense and used as unit coördinate vectors we have

$$
a=I_{1}, \bar{a}=I_{6}, b=I_{2}, \bar{b}=I_{5}, c=I_{3}, \bar{c}=I_{4} .
$$

Since a six-vector has six independent components and a scalar product of the form (5.4), its components may be interpreted as those of a vector in six-dimensional euclidean space. When the unit coördinate vectors in $V_{4}$ are rotated the transformation of the components of a six-vector $C$ are of the type (4.9), or

$$
\begin{array}{ll}
C_{1}{ }^{\prime}=C_{1}, & C_{6}{ }^{\prime}=C_{6}, \\
C_{2}{ }^{\prime}=C_{2} \cos \theta-C_{4} \sin \theta, & C_{4}{ }^{\prime}=C_{2} \sin \theta+C_{4} \cos \theta, \\
C_{5}{ }^{\prime}=C_{5} \cos \theta-C_{3} \sin \theta, & C_{3}{ }^{\prime}=C_{5} \sin \theta+C_{3} \cos \theta .
\end{array}
$$

But under these transformations not only the scalar product of two sixvectors is invariant, but also the meaning of the dual is preserved. Only those special rotations of the coördinate axes in six-dimensional space which yield transformations of the type (5.10) are permitted while considering our sixvectors as vectors of this space.

6. Three-vectors. In addition to the elementary six-vector there is another special type of six-vector which is very useful for our purpose; it is called a three-vector. ${ }^{*}$ If $a$ is a unit elementary six-vector, the six-vector

$$
A=\lambda a+\mu \bar{a}
$$

is called a three-vector if the characteristic numbers satisfy the condition

$$
\lambda^{2}=\mu^{2} .
$$

If $\lambda=\mu$ then $A$ is self-dual: $A=\bar{A}$; hence $A_{s}=A_{7-s}(s=1,2, \cdots, 6)$ and the components of $A$ can be written

$$
\left(A_{1}, A_{2}, A_{3}, A_{3}, A_{2}, A_{1}\right) \text {. }
$$

\footnotetext{
* These vectors were introduced in the form used here by Rainich, Les indices dans un champ de tenseurs, Comptes Rendus, vol. 185 (1927), p. 1009.
} 
Likewise if $\lambda=-\mu$ for the six-vector $B=\lambda b+\mu \bar{b}$, then $B$ is anti-self-dual: $B=-\bar{B}$, and its components are

$$
\left(B_{1}, B_{2}, B_{3},-B_{3},-B_{2},-B_{1}\right) \text {. }
$$

It follows from (6.2) and (6.3) that each three-vector of one type is perpendicular to every three-vector of the opposite type:

$$
A B=0 \text { if } A=\bar{A}, B=-\bar{B} \text {. }
$$

Furthermore if $C$ and $D$ are two three-vectors of the same type then their scalar product can be written

$$
C D=2\left(C_{1} D_{1}+C_{2} D_{2}+C_{3} D_{3}\right) .
$$

The numbers $C_{1}, C_{2}, C_{3}$ may be considered as the components of a vector in three-dimensional euclidean space, and the same statement holds for $D_{1}, D_{2}$, $D_{3}$, for these numbers transform so that the expression $C_{1} D_{1}+C_{2} D_{2}+C_{3} D_{3}$ remains invariant.

III. The Two Parts of THE Riemann TENSOR AS FUnCtions OF SIX-Vectors

7. The Riemann tensor as a function of elementary six-vectors. The expression (3.2), which the Riemann tensor assumes when referred to our local coördinate system, can be written

$$
4 R_{\alpha \beta, \gamma \delta} x_{\alpha} y_{\beta} u_{\gamma} v_{\delta}=R_{\alpha \beta, \gamma \delta}\left|\begin{array}{cc}
x_{\alpha} & x_{\beta} \\
y_{\alpha} & y_{\beta}
\end{array}\right|\left|\begin{array}{cc}
u_{\gamma} & u_{\delta} \\
v_{\gamma} & v_{\delta}
\end{array}\right| .
$$

These determinants are the components of the elementary six-vectors $a$ and $b$ determined by $x, y$ and $u, v$ respectively so that

$$
4 R(x, y ; u, v)=R_{\alpha \beta, \gamma \delta} a_{\alpha \beta} b_{\gamma \delta} .
$$

When the single subscripts are used for the components of $a$ and $b$, and when the pairs $m n$ and $p q$ of subscripts in $R_{m n, p q}$ are replaced by the corresponding numbers in table (5.2), an examination of the sum on the right shows that the last equation can be written

$$
R(x, y ; u, v)=R(a, b)=R_{\rho \sigma} a_{\rho} b_{\sigma}(\rho, \sigma=1,2, \cdots, 6) .
$$

It is well to repeat that the new symbols $R_{s t}$ are defined according to (5.2):

$$
R_{11}=R_{12,12}, R_{12}=R_{12,13}, \cdots, R_{56}=R_{42,34}, R_{66}=R_{34,34} .
$$

Since $R_{12,12}=R(i, j ; i, j)$ then $R_{11}=R\left(I_{1}, I_{1}\right) ; R_{s t}$ are the numbers which $R(a, b)$ assigns to the unit coördinate six-vectors:

$$
R_{s t}=R\left(I_{s}, I_{t}\right) \quad(s, t=1,2, \cdots, 6) .
$$


The Riemann tensor is therefore a function of two elementary six-vectors, the function being bilinear in their components. This statement includes the linearity properties (3.1), but the second of these can be written

$$
R(\rho a, b)=\rho R(a, b),
$$

where $\rho$ is any number. The symmetry property (3.5) becomes

$$
R(a, b)=R(b, a),
$$

and the anti-symmetry property (3.4) is included in (7.4) for $\rho=-1$.

The cyclic property (3.9) can be written

$$
R_{16}+R_{25}+R_{34}=R\left(I_{1}, I_{6}\right)+R\left(I_{2}, I_{5}\right)+R\left(I_{3}, I_{4}\right)=0 .
$$

In terms of the new components, (7.5) and (3.12) take the forms

$$
\begin{aligned}
R_{s t} & =R_{t s} & (s, t & =1,2, \cdots, 6), \\
R & =2 R_{p \rho}=2 R\left(I_{\rho}, I_{\rho}\right) & (\rho & =1,2, \cdots, 6) .
\end{aligned}
$$

8. The two parts of $R(a, b)$. The two parts into which the Riemann tensor is decomposed here, and the properties of these parts, are not new.* The method of obtaining these parts and their properties, however, is simplified by using six-vectors.

The identity

$$
R(a, b)=[R(a, b)+R(\bar{a}, \bar{b})] / 2+[R(a, b)-R(\bar{a}, \bar{b})] / 2
$$

expresses the Riemann tensor as the sum of the two functions

$$
\begin{aligned}
& G(a, b)=[R(a, b)+R(\bar{a}, \bar{b})] / 2, \\
& E(a, b)=[R(a, b)-R(\bar{a}, \bar{b})] / 2 .
\end{aligned}
$$

The identity can now be written

$$
R(a, b)=G(a, b)+E(a, b) .
$$

In terms of components (8.1) becomes

$$
\begin{aligned}
G(a, b) & =\left(R_{\rho \sigma} a_{\rho} b_{\sigma}+R_{\rho \sigma} \bar{a}_{\rho} \bar{b}_{\sigma}\right) / 2 \\
& =\left(R_{\rho \sigma} a_{\rho} b_{\sigma}+R_{7-\rho, 7-\sigma} \bar{a}_{7-\rho} \bar{b}_{7-\sigma}\right) / 2 \\
& =\left(R_{\rho \sigma}+R_{7-\rho, 7-\sigma}\right) a_{\rho} b_{\sigma} / 2 \quad(\rho, \sigma=1,2, \cdots, 6) .
\end{aligned}
$$

Hence $G(a, b)$ is a bilinear function of the components of two elementary six-vectors and it follows that it is a fourth-rank tensor.

* Rainich, Electricity in curved space-time, Nature, vol. 115 (1925), p. 498; Cartan, Variétés d connexion affine, Annales de l'Ecole Normale, vol. 42 (1925), p. 87; Einstein, Über die formale Beziehung des Riemannschen Krümmungstensors zu den Feldgleichungen der Gravitation, Mathematische Annalen, vol. 97 (1926), p. 99. 
According to (8.1) $G(a, b)$ has the properties (7.4), (7.5) and (7.6) of $R(a, b)$, and an additional property that its value is unchanged when its arguments are replaced by their duals. Hence $G(a, b)$, or the tensor of the first type, has all the fundamental properties of the Riemann tensor together with the property

$$
G(\bar{a}, \bar{b})=G(a, b) .
$$

Likewise the second part $E(a, b)$, or the tensor of the second type, has all the fundamental properties of the Riemann tensor and the additional property

$$
E(\bar{a}, \bar{b})=-E(a, b) .
$$

In terms of components (8.4) can be written

$$
G_{s t}=G_{7-8,7-t} \quad(s, t=1,2, \cdots, 6) .
$$

Properties (8.6) and (7.7) show that the sixth-order determinant of the components $G_{s t}$ is symmetric to both diagonals, so in view of the cyclic property (7.6) the number of independent components of the tensor of the first type is reduced to eleven.

Similarly

$$
E_{s t}=-E_{7-8,7-t} \quad(s, t=1,2, \cdots, 6),
$$

and the number of independent components of $E(a, b)$ is reduced to nine. According to (8.7) all components involved in the cyclic property vanish, and also the second contracted tensor of $E(a, b)$ vanishes so that by twice contracting both members of (8.3) we find

$$
R=G=4\left(G_{11}+G_{22}+G_{33}\right) .
$$

We shall now proceed with our problem by referring each of the parts $G(a, b)$ and $E(a, b)$ separately to their intrinsic directions.

IV. The TENSOR OF THE FIRST TYPE

9. A generalization of $G(a, b)$; the function $G(A)$. It was shown above that the tensor of the first type has the properties

$$
\begin{aligned}
& G(a, b)=G_{\rho \sigma} a_{\rho} b_{\sigma} \quad(\rho, \sigma=1,2, \cdots, 6), \\
& G(a, b)=G(b, a), \quad G(\bar{a}, \bar{b})=G(a, b),
\end{aligned}
$$

and also the cyclic property (7.6)

$G(a, b)$ was defined above when its arguments are elementary six-vectors. We now define it when its arguments are any six-vectors by requiring it to have the same formal properties and reduce to the tensor of the first type 
when its arguments are elementary. $G(A, B)$ is then a scalar function of $A, B$ which has the properties

$$
\begin{aligned}
G(A+C, B) & =G(A, B)+G(C, B), \\
G(\gamma A, B) & =\gamma G(A, B),
\end{aligned}
$$

where $\gamma$ is any number, and

$$
G(A, B)=G(B, A), G(\bar{A}, \bar{B})=G(A, B) ;
$$

it also has the cyclic property.

When $A$ and $B$ are expressed in terms of the unit coördinate six-vectors and the linearity properties (9.2) are applied, we find

$$
G(A, B)=G_{\rho \sigma} A_{\rho} B_{\sigma} \quad(\rho, \sigma=1,2, \cdots, 6),
$$

where the coefficients $G_{s t}=G\left(I_{s}, I_{t}\right)$ are the components of the tensor of the first type.

According to the definition of $G(A, B)$ the expression $G_{\rho \sigma} A_{\rho} B_{\sigma}$ is an invariant, and it is readily shown from this and Theorem 2 that $G_{\rho p} A_{\rho}$ are the components of a six-vector. We call this six-vector $G(A)$ and denote its components by $G_{p}(A)$ :

$$
G_{p}(A)=G_{\rho p} A_{\rho} \quad(\rho, p=1,2, \cdots, 6) .
$$

$G(A)$ is a function which assigns a six-vector to its argument $A$. The relation between this vector function and the scalar function follows from (9.4):

$$
G(A, B)=G_{\sigma}(A) B_{\sigma}=G(A) B \quad(\sigma=1,2, \cdots, 6),
$$

where $G(A) B$ is the scalar product of the six-vectors $G(A)$ and $B$.

Now (9.6) enables us to express the properties of $G(A, B)$ in terms of the function $G(A)$. For example, the second property in (9.3) can be written

$$
G_{\sigma}(\bar{A}) \bar{B}_{\sigma}=G_{\sigma}(A) B_{\sigma} \quad(\sigma=1,2, \cdots, 6),
$$

but the summation for $7-\sigma$ is the same as for $\sigma$, so

$$
G_{\sigma}(\bar{A}) B_{7-\sigma}=G_{7-\sigma}(A) B_{7-\sigma} \quad(\sigma=1,2, \cdots, 6) .
$$

This is an identity in the components $B_{p}$ and hence

$$
G_{p}(\bar{A})=G_{7-p}(A) \quad(p=1,2, \cdots, 6),
$$

which means that the vectors $G(\bar{A})$ and $G(A)$ are duals of each other. If $\bar{G}(A)$ denotes the dual of $G(A)$, then

$$
G(\bar{A})=\bar{G}(A) .
$$

The other properties of $G(A)$ in the set 


$$
\begin{aligned}
G(A+B) & =G(A)+G(B), & G(\gamma A) & =\gamma G(A), \\
G(A) B & =G(B) A, & G(\bar{A}) & =\bar{G}(A)
\end{aligned}
$$

are found in like manner from (9.2), (9.3) and (9.6). Hence $G(A)$ is a symmetric linear six-vector function which has the property that the vector which it assigns to $\bar{A}$ is the dual of the vector which it assigns to $A$. Furthermore, $G(A, B)$ satisfies the cyclic property, so that

$$
G\left(I_{1}\right) I_{6}+G\left(I_{2}\right) I_{5}+G\left(I_{3}\right) I_{4}=G_{16}+G_{25}+G_{34}=0 .
$$

10. Principal directions of a symmetric linear vector function in a $V_{n}$. In the two sections following this we need convenient references to the geometry of symmetric linear vector functions in euclidean spaces of six and three dimensions, and in $\$ 18$ we refer to the geometry of a symmetric tensor of rank two in a $V_{4}$. Consequently in this section we review the known* geometry of the symmetric linear vector function and symmetric second-rank tensor at a point of a $V_{n}$ with positive definite fundamental quadratic form.

The components of vectors and tensors are referred here to a rectangular locally cartesian coördinate system at the point. Let $P$ and $Q$ be any two vectors at this point and let $f_{r s}$ be the components of a symmetric tensor,

$$
f(P, Q)=f_{\rho \sigma} P_{\rho} Q_{\sigma}=f(Q, P) \quad(\rho, \sigma=1,2, \cdots, n),
$$

of the second rank. The functions

$$
f_{r}(P)=f_{\rho r} P_{\rho} \quad(\rho, r=1,2, \cdots, n)
$$

are the $n$ components of a symmetric linear vector function $f(P)$, and according to (10.1) the tensor $f(P, Q)$ is the scalar product of $f(P)$ and $Q$,

$$
f(P, Q)=f_{\sigma}(P) Q_{\sigma}=f(P) Q \quad(\sigma=1,2, \cdots, n) .
$$

If $P$ and $f(P)$ have the same direction,

$$
f(P)=\omega P,
$$

then $P$ belongs to an invariable direction, or principal direction, of $f(P)$ with $\omega$ as multiplier. This condition (10.3) is given by the $n$ scalar equations

$$
f_{\rho r} P_{\rho}-\omega P_{r}=0 \quad(\rho, r=1,2, \cdots, n),
$$

and this system has solutions other than $P_{r}=0$ if $\omega$ satisfies

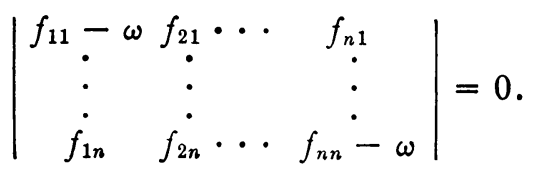

* See Struik, Grundzüge der mehrdimensionalen Differentialgeometrie, 1922, p. 33, or Eisenhart, these Transactions, vol. 25 (1923), p. 259. 
This determinant is symmetric to its principal diagonal and in this case the characteristic equation (10.4) has only real roots.

If these roots $\omega_{1}, \omega_{2}, \cdots, \omega_{n}$ are distinct then $f(P)$ has just $n$ mutually perpendicular invariable directions, each with one of the roots as a multiplier. If a root has a multiplicity $m$, there is an $m$-space each direction of which is invariable with this root as a multiplier. This $m$-space is perpendicular to the principal directions or spaces corresponding to the other roots. Hence if $m$ mutually perpendicular directions are chosen in each principal $m$-space, then regardless of multiplicity of the roots, there is always at least one orthogonal set of $n$ invariable directions of $f(P)$.

Let unit coördinate vectors $W$, be taken along these $n$ invariable directions and let $P_{r}$ be the components of $P$ relative to $W_{r}$. Then due to the linearity of $f(P)$ we have

$$
f(P)=f\left(P_{\sigma} W_{\sigma}\right)=P_{\sigma} f\left(W_{\sigma}\right) \quad(\sigma=1,2, \cdots, n),
$$

and if $\omega_{r}$ are the multipliers of $W_{r}$, this becomes

$$
f(P)=\omega_{\sigma} P_{\sigma} W_{\sigma} \quad(\sigma=1,2, \cdots, n) .
$$

It follows from (10.2) that

$$
f(P, Q)=\omega_{\sigma} P_{\sigma} Q_{\sigma} \quad(\sigma=1,2, \cdots, n),
$$

where the components $Q_{r}$ are also referred to the unit vectors $W_{r}$. Hence the symmetric linear vector function and the symmetric second-rank tensor are determined by an orthogonal set of $n$ intrinsic directions and $n$ numbers, one corresponding to each direction.

11. Invariable directions of $G(A)$. We have seen that $G(A)$ is a symmetric linear vector function of the six-vector $A$, and since $A$ can be considered as a vector of a six-dimensional euclidean space it follows that $G(A)$ can be considered as a symmetric linear vector function in this space. According to the foregoing section then, there is at least one set of six mutually prependicular invariable directions for $G(A)$ whose multipliers are the roots of the characteristic equation

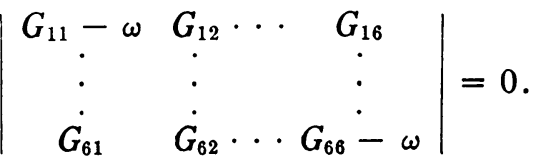

According to (9.7),

$$
G(\bar{B})=\bar{G}(B)
$$


Now if $B$ is a vector of invariable direction corresponding to a root $\alpha$ of (11.1) then

$$
G(B)=\alpha B,
$$

and it follows that the duals of $G(B)$ and $\alpha B$ are equal:

$$
\bar{G}(B)=\alpha \bar{B} \text {. }
$$

When (11.2) is applied to the left-hand member, this equation becomes

$$
G(\bar{B})=\alpha \bar{B} .
$$

Hence the dual of each vector of invariable direction of $G(A)$ is also a vector of invariable direction; the multiplier is the same for both directions.

ThEOREM 5. Each multiplier of an invariable direction of $G(A)$ is the multiplier of at least one invariable direction whose vectors are three-vectors.

To prove this let $B$ be a vector of invariable direction with multiplier $\alpha$. Then $B$ satisfies (11.3) and (11.4) and hence

$$
G(B+\bar{B})=G(B)+G(\bar{B})=\alpha(B+\bar{B}) ;
$$

that is, $B+\bar{B}$ belongs to an invariable direction with $\alpha$ as multiplier. But $B+\bar{B}$ is self-dual, so it is a three-vector and the theorem is proved. This proof fails if $B=-\bar{B}$, but in this case $B$ itself satisfies the conditions of the theorem.

If the six roots of (11.1) are distinct then there are just six mutually perpendicular invariable directions each having one of these roots as multiplier. It follows from Theorem 5 that in this case the vectors of each of these directions are three-vectors.

12. $G(A)$ and three-vectors. To three-vectors of one type $G(A)$ assigns three-vectors of the same type, for if $A=\bar{A}$, then, according to (11.2).

$$
G(A)=G(\bar{A})=\bar{G}(A) \text {. }
$$

It follows in like manner that when its argument is anti-self-dual, the function is anti-self-dual. Now since all three-vectors of the one type can be considered as the vectors of a three-dimensional euclidean space, the function $G(A)$ can be considered as a symmetric linear vector function in this space when $A$ is a three-vector of this type. Consequently in the application of this function to self-dual three-vectors $G(A)$ has at least three mutually perpendicular invariable directions whose vectors are self-dual three-vectors (cf. \$10). Likewise it has at least three mutually perpendicular invariable directions whose vectors are anti-self-dual.

Let $A^{\prime}, B^{\prime}, C^{\prime}$ be self-dual three-vectors along the invariable directions of the first type and let the square of each be 2 . Let $\alpha^{\prime}, \beta^{\prime}, \gamma^{\prime}$ be their respec- 
tive multipliers. Likewise let $A^{\prime \prime}, B^{\prime \prime}, C^{\prime \prime}$ denote anti-self-dual three-vectors with square 2 taken along the invariable directions of the second type, with $\alpha^{\prime \prime}, \beta^{\prime \prime}, \gamma^{\prime \prime}$ as the corresponding multipliers. Since the product of any two sixvectors of opposite types is zero, each invariable direction of the first type is perpendicular to those of the second type, so these six directions are mutually perpendicular. We can now write

$$
\begin{aligned}
& G\left(A^{\prime}\right)=\alpha^{\prime} A^{\prime}, \quad G\left(B^{\prime}\right)=\beta^{\prime} B^{\prime}, \quad G\left(C^{\prime}\right)=\gamma^{\prime} C^{\prime}, \\
& G\left(A^{\prime \prime}\right)=\alpha^{\prime \prime} A^{\prime \prime}, \quad G\left(B^{\prime \prime}\right)=\beta^{\prime \prime} B^{\prime \prime}, \quad G\left(C^{\prime \prime}\right)=\gamma^{\prime \prime} C^{\prime \prime},
\end{aligned}
$$

where

$$
\begin{gathered}
A^{\prime} B^{\prime}=A^{\prime} C^{\prime}=A^{\prime} A^{\prime \prime}=\cdots=C^{\prime} C^{\prime \prime}=0 \\
A^{\prime 2}=B^{\prime 2}=\cdots=C^{\prime \prime 2}=2, \\
A^{\prime}=\bar{A}^{\prime}, B^{\prime}=\bar{B}^{\prime}, C^{\prime}=\bar{C}^{\prime} ; A^{\prime \prime}=-\bar{A}^{\prime \prime}, B^{\prime \prime}=-\bar{B}^{\prime \prime}, C^{\prime \prime}=-\bar{C}^{\prime \prime} .
\end{gathered}
$$

Each of the six-multipliers $\alpha^{\prime}, \beta^{\prime}, \cdots, \gamma^{\prime \prime}$ must be a root of the sixthdegree characteristic equation (11.1) for these roots are the only multipliers of invariable directions of $G(A)$. Moreover these six-multipliers are the only multipliers of three-vector invariable directions, so it follows from Theorem 5 that each of the roots of (11.1) belongs to the set of multipliers $\alpha^{\prime}, \beta^{\prime}, \cdots, \gamma^{\prime \prime}$. Therefore this set of multipliers is identical to the set of roots of (11.1), and the following theorem is established.

THEOREM 6. For the function $G(A)$, there is always at least one set of six mutually perpendicular invariable directions such that vectors along three of them are self-dual and vectors along the other three are anti-self-dual. The multipliers of these directions are the roots of (11.1) and if these roots are distinct, there is just one set of invariable directions.

13. Intrinsic directions whose vectors are elementary six-vectors. From the mutually perpendicular three-vectors

$$
A^{\prime}, B^{\prime}, C^{\prime}, \quad A^{\prime \prime}, B^{\prime \prime}, C^{\prime \prime}
$$

used in (12.1), let us form the following new set of six-vectors:

$$
\begin{aligned}
& a=\left(A^{\prime}+A^{\prime \prime}\right) / 2, \quad b=\left(B^{\prime}+B^{\prime \prime}\right) / 2, \quad c=\left(C^{\prime}+C^{\prime \prime}\right) / 2, \\
& \bar{a}=\left(A^{\prime}-A^{\prime \prime}\right) / 2, \quad \bar{b}=\left(B^{\prime}-B^{\prime \prime}\right) / 2, \quad \bar{c}=\left(C^{\prime}-C^{\prime \prime}\right) / 2 .
\end{aligned}
$$

As a consequence of Theorem 1 and (12.2), (12.3), (12.4), these vectors $a, b, c$ and $\bar{a}, \bar{b}, \bar{c}$ are mutually perpendicular unit elementary six-vectors, so they form a set of unit coördinate six-vectors (Theorem 4 ):

$$
I_{1}=a, I_{2}=b, I_{3}=c, I_{4}=\bar{c}, I_{5}=\bar{b}, I_{6}=\bar{a} .
$$


From the three-vectors (13.1) five other sets of intrinsic coördinate sixvectors can be found, the vectors in each set being determined as in (13.2) by pairs of these three-vectors so that each pair consists of a self-dual and an anti-self-dual three-vector. These six sets of coördinate six-vectors which are intrinsically related to $G(A)$ are called elementary six-vector skeletons of $G(A)$. When (11.1) has distinct roots there are just six of these skeletons; the relations between them are discussed later.

Let us see how $G(A)$ operates on, or transforms, the vectors of its skeleton. It follows from (13.2) and (13.3) that

$$
\begin{aligned}
& A^{\prime}=I_{1}+I_{6}, \quad B^{\prime}=I_{2}+I_{5}, \quad C^{\prime}=I_{3}+I_{4}, \\
& A^{\prime \prime}=I_{1}-I_{6}, \quad B^{\prime \prime}=I_{2}-I_{5}, \quad C^{\prime \prime}=I_{3}-I_{4} .
\end{aligned}
$$

When our function is applied to $I_{1}$, we find

$$
G\left(I_{1}\right)=G\left(A^{\prime}\right) / 2+G\left(A^{\prime \prime}\right) / 2 \text {; }
$$

but $A^{\prime}$ and $A^{\prime \prime}$ belong to invariable directions, so

$$
G\left(I_{1}\right)=\alpha^{\prime} A^{\prime} / 2+\alpha^{\prime \prime} A^{\prime \prime} / 2=\left(\alpha^{\prime}+\alpha^{\prime \prime}\right) I_{1} / 2+\left(\alpha^{\prime}-\alpha^{\prime \prime}\right) I_{6} / 2 .
$$

The transformations of all six unit vectors can be written

$$
\begin{array}{lll}
G\left(I_{1}\right)=\alpha I_{1}+\rho I_{6}, & G\left(I_{2}\right)=\beta I_{2}+\sigma I_{5}, & G\left(I_{3}\right)=\gamma I_{3}+\delta I_{4}, \\
G\left(I_{6}\right)=\rho I_{1}+\alpha I_{6}, & G\left(I_{5}\right)=\sigma I_{2}+\beta I_{5}, & G\left(I_{4}\right)=\delta I_{3}+\gamma I_{4},
\end{array}
$$

where the new numbers represent the following combinations of the roots of (11.1):

$$
\begin{array}{lll}
\alpha=\left(\alpha^{\prime}+\alpha^{\prime \prime}\right) / 2, & \beta=\left(\beta^{\prime}+\beta^{\prime \prime}\right) / 2, & \gamma=\left(\gamma^{\prime}+\gamma^{\prime \prime}\right) / 2, \\
\rho=\left(\alpha^{\prime}-\alpha^{\prime \prime}\right) / 2, & \sigma=\left(\beta^{\prime}-\beta^{\prime \prime}\right) / 2, & \delta=\left(\gamma^{\prime}-\gamma^{\prime \prime}\right) / 2 .
\end{array}
$$

The roots of the characteristic equation of $G(A)$ are not independent. For when both members of the first equation of (13.5) are scalarly multiplied by $I_{1}$, we get

$$
G\left(I_{1}\right) I_{1}=\alpha ;
$$

but this is the component $G_{11}$ of the tensor of the first type, referred to the intrinsic coördinate six-vectors. In view of (13.5), all of the non-vanishing components of this tensor can be given in terms of the six components

$$
G_{11}=\alpha, G_{22}=\beta, G_{33}=\gamma, G_{16} \doteq \rho, G_{25}=\sigma, G_{34}=\delta .
$$

The cyclic property (9.8) now becomes

$$
\rho+\sigma+\delta=0 \text {; }
$$

and in terms of the roots of the characteristic equation this becomes

$$
\alpha^{\prime}+\beta^{\prime}+\gamma^{\prime}=\alpha^{\prime \prime}+\beta^{\prime \prime}+\gamma^{\prime \prime} \text {. }
$$


Hence the sum of the multipliers of the three invariable directions of one type equals the sum of the multipliers of the invariable directions of the opposite type.

From (13.7) and (8.8) it follows that the second contracted Riemann tensor is given by

$$
R=G=4(\alpha+\beta+\gamma) .
$$

14. Six-vector geometry of the tensor of the first type. If $d$ and $m$ are any two elementary six-vectors, the tensor of the first type is given by

$$
G(d, m)=G(d) m \text {. }
$$

Let $d_{s}$ be the components of $d$ relative to the coördinate six-vectors (13.3) of the skeleton of $G(A)$; then

$$
d=d_{\rho} I_{\rho} \quad(\rho=1,2, \cdots, 6)
$$

and when the argument of $G(d)$ is so written we find, according to (13.5),

$$
\begin{aligned}
G(d)= & \alpha\left(d_{1} I_{1}+d_{6} I_{6}\right)+\beta\left(d_{2} I_{2}+d_{5} I_{6}\right)+\gamma\left(d_{3} I_{3}+d_{4} I_{4}\right) \\
& +\rho\left(d_{1} I_{6}+d_{6} I_{1}\right)+\sigma\left(d_{2} I_{5}+d_{5} I_{2}\right)+\delta\left(d_{3} I_{4}+d_{4} I_{3}\right) .
\end{aligned}
$$

This can be put in the more convenient form

$$
G(d)=R d / 12+\alpha_{\rho}\left(d_{\rho} I_{\rho}+d_{7-\rho} I_{7-\rho}\right)+\beta_{\rho}\left(d_{\rho} I_{7-\rho}+d_{7-\rho} I_{\rho}\right)(\rho=1,2,3),
$$

where $R$ is the second contracted Riemann tensor and

$$
\begin{array}{lll}
\alpha_{1}=\alpha-R / 12, & \alpha_{2}=\beta-R / 12, & \alpha_{3}=\gamma-R / 12, \\
\beta_{1}=\rho, & \beta_{2}=\sigma, & \beta_{3}=\delta .
\end{array}
$$

It follows from (13.10) and (13.8) that

$$
\alpha_{1}+\alpha_{2}+\alpha_{3}=\beta_{1}+\beta_{2}+\beta_{3}=0 .
$$

In view of (14.1) our results can be applied at once to $G(d, m)$ :

THEOREM 7. The tensor of the first type is determined by an intrinsic set of unit coördinate six-vectors and seven numbers, six of which correspond to these vectors. Because of (14.6) only five of these numbers are independent. There are at least six of these intrinsic coördinate systems and when referred to one of them the tensor takes the form

$$
\begin{array}{r}
G(d, m)=d m R / 12+\alpha_{\rho}\left(d_{\rho} m_{\rho}+d_{7-\rho} m_{7-\rho}\right)+\beta_{\rho}\left(d_{\rho} m_{7-\rho}+d_{7-\rho} m_{\rho}\right) \\
(\rho=1,2,3),
\end{array}
$$

where

$$
\alpha_{1}+\alpha_{2}+\alpha_{3}=\beta_{1}+\beta_{2}+\beta_{3}=0 .
$$

The part $d m R / 12$ of this tensor is independent of a skeleton. 
15. Four-vector geometry of the tensor of the first type. Let $x, y$ and $u$, $v$ be two pairs of four-vectors which determine $d$ and $m$. The tensor $d m R / 12$ can be expressed as a determinant involving $x, y, u, v$ according to (5.1). It is denoted below by $H(x, y ; u, v)$ and the remaining part of the tensor of the first type is called $F(x, y ; u, v)$.

According to Theorem 4 the intrinsic coördinate six-vectors found above determine a set of coördinate four-vectors $i, j, k, l$ so that the $i, j$ plane is the plane of $I_{1}$, the $i, k$ plane is that of $I_{2}$, etc. These four-vectors are intrinsically related to the tensor of the first type, and the set of four mutually perpendicular directions determined by them is called a skeleton of this tensor.

Let the components of $x, y, u, v$ be referred to this intrinsic coördinate system, and let the components of $d$ and $m$ be given in terms of these components of $x, y, u, v$ according to (4.1) and (5.2). Then (14.7) enables us to describe the tensor of the first type in terms of its skeleton and its four-vector arguments by means of the following equations:

$$
G(x, y ; u, v)=H(x, y ; u, v)+F(x, y ; u, v)
$$

where

$$
H(x, y ; u, v)=\left|\begin{array}{ll}
x u & x v \\
y u & y v
\end{array}\right| R / 12
$$

and where

$$
\begin{aligned}
& F(x, y ; u, v)= \\
& \alpha_{1}\left(\left|\begin{array}{ll}
x_{1} & x_{2} \\
y_{1} & y_{2}
\end{array}\right|\left|\begin{array}{ll}
u_{1} & u_{2} \\
v_{1} & v_{2}
\end{array}\right|+\left|\begin{array}{ll}
x_{3} & x_{4} \\
y_{3} & y_{4}
\end{array}\right|\left|\begin{array}{ll}
u_{3} & u_{4} \\
v_{3} & v_{4}
\end{array}\right|\right) \\
& +\beta_{1}\left(\left|\begin{array}{ll}
x_{1} & x_{2} \\
y_{1} & y_{2}
\end{array}\right|\left|\begin{array}{ll}
u_{3} & u_{4} \\
v_{3} & v_{4}
\end{array}\right|+\left|\begin{array}{ll}
x_{3} & x_{4} \\
y_{3} & y_{4}
\end{array}\right|\left|\begin{array}{ll}
u_{1} & u_{2} \\
v_{1} & v_{2}
\end{array}\right|\right) \\
& +\alpha_{2}\left(\left|\begin{array}{ll}
x_{1} & x_{3} \\
y_{1} & y_{3}
\end{array}\right|\left|\begin{array}{ll}
u_{1} & u_{3} \\
v_{1} & v_{3}
\end{array}\right|+\left|\begin{array}{ll}
x_{4} & x_{2} \\
y_{4} & y_{2}
\end{array}\right|\left|\begin{array}{ll}
u_{4} & u_{2} \\
v_{4} & v_{2}
\end{array}\right|\right) \\
& +\beta_{2}\left(\left|\begin{array}{ll}
x_{1} & x_{3} \\
y_{1} & y_{3}
\end{array}\right|\left|\begin{array}{ll}
u_{4} & u_{2} \\
v_{4} & v_{2}
\end{array}\right|+\left|\begin{array}{ll}
x_{4} & x_{2} \\
y_{4} & y_{2}
\end{array}\right|\left|\begin{array}{ll}
u_{1} & u_{3} \\
v_{1} & v_{3}
\end{array}\right|\right) \\
& +\alpha_{3}\left(\left|\begin{array}{ll}
x_{1} & x_{4} \\
y_{1} & y_{4}
\end{array}\right|\left|\begin{array}{ll}
u_{1} & u_{4} \\
v_{1} & v_{4}
\end{array}\right|+\left|\begin{array}{ll}
x_{2} & x_{3} \\
y_{2} & y_{3}
\end{array}\right|\left|\begin{array}{ll}
u_{2} & u_{3} \\
v_{2} & v_{3}
\end{array}\right|\right) \\
& +\beta_{3}\left(\left|\begin{array}{ll}
x_{1} & x_{4} \\
y_{1} & y_{4}
\end{array}\right|\left|\begin{array}{ll}
u_{2} & u_{3} \\
v_{2} & v_{3}
\end{array}\right|+\left|\begin{array}{ll}
x_{2} & x_{3} \\
y_{2} & y_{3}
\end{array}\right|\left|\begin{array}{ll}
u_{1} & u_{4} \\
v_{1} & v_{4}
\end{array}\right|\right)
\end{aligned}
$$


The coefficients are related according to (14.6), viz.,

$$
\alpha_{1}+\alpha_{2}+\alpha_{3}=\beta_{1}+\beta_{2}+\beta_{3}=0,
$$

and our results can be summarized as follows.

THEOREM 8. The tensor $F(x, y ; u, v)$ is determined by a skeleton and four independent numbers. Each of the three numbers $\alpha_{1}, \alpha_{2}, \alpha_{3}$ is associated with a different pair of absolutely perpendicular planes of the skeleton, $\alpha_{1}$, with the $i, j$ and $k, l$ planes, etc. The same is true of the numbers $\beta_{1}, \beta_{2}, \beta_{3}$. The tensor $H(x, y ; u, v)$ depends only upon the number $R$, so the tensor of the first type is determined by a skeleton and five independent numbers.

By referring the tensor of the first type to its skeleton its independent components have been reduced in number from eleven to five. The scheme of its components can be written from (14.7) or from the formulas of this section.

16. Relations between skeletons. Our intrinsic coördinate six-vectors (13.3) were determined by taking sums and differences of pairs of three-vectors of invariable direction for $G(A)$. These pairs consisted of one member from the group $A^{\prime}, B^{\prime}, C^{\prime}$ of self-dual vectors and the other from the group $A^{\prime \prime}$, $B^{\prime \prime}, C^{\prime \prime}$ of anti-self-dual vectors. Out of the six possible sets of pairs, however, we used only the set

$$
\begin{aligned}
& I_{1}=\left(A^{\prime}+A^{\prime \prime}\right) / 2, \quad I_{2}=\left(B^{\prime}+B^{\prime \prime}\right) / 2, \quad I_{3}=\left(C^{\prime}+C^{\prime \prime}\right) / 2, \\
& I_{6}=\left(A^{\prime}-A^{\prime \prime}\right) / 2, \quad I_{5}=\left(B^{\prime}-B^{\prime \prime}\right) / 2, \quad I_{4}=\left(C^{\prime}-C^{\prime \prime}\right) / 2 .
\end{aligned}
$$

Now consider the set of coördinate six-vectors obtained as follows:

$$
\begin{aligned}
& I_{1}^{\prime}=I_{1}=\left(A^{\prime}+A^{\prime \prime}\right) / 2, I_{2}^{\prime}=\left(B^{\prime}+C^{\prime \prime}\right) / 2, I_{3}^{\prime}=\left(C^{\prime}-B^{\prime \prime}\right) / 2 \\
& I_{6}^{\prime}=I_{6}=\left(A^{\prime}-A^{\prime \prime}\right) / 2, I_{6}^{\prime}=\left(B^{\prime}-C^{\prime \prime}\right) / 2, I_{4}^{\prime}=\left(C^{\prime}+B^{\prime \prime}\right) / 2 .
\end{aligned}
$$

The new set of intrinsic coördinate four-vectors are related to these new coordinate six-vectors in the usual way: $i^{\prime}$ and $j^{\prime}$ determine $I_{1}^{\prime}, i^{\prime}$ and $k^{\prime}$ determine $I_{2}^{\prime}, \cdots, k^{\prime}$ and $l^{\prime}$ determine $I_{6}{ }^{\prime}$. It follows from (16.1) and (16.2) that

$$
\begin{aligned}
& I_{1}^{\prime}=I_{1}, 2 I_{2}^{\prime}=I_{2}+I_{3}-I_{4}+I_{5}, 2 I_{3}^{\prime}=-I_{2}+I_{3}+I_{4}+I_{5}, \\
& I_{6}^{\prime}=I_{6}, 2 I_{5}^{\prime}=I_{2}-I_{3}+I_{4}+I_{5}, 2 I_{4}^{\prime}=I_{2}+I_{3}+I_{4}-I_{5} .
\end{aligned}
$$

If $x$ and $y$ are two four-vectors which determine an elementary six-vector $b$ and if $w$ and $z$ are any two unit coördinate four-vectors, then according to the definition of the components of $b$, the quantity

$$
\left|\begin{array}{ll}
x w & x z \\
y w & y z
\end{array}\right|
$$

is the component of $b$ with respect to the coördinate plane of $w$ and $z$. 
Hence the equation

$$
2 I_{2}^{\prime}=I_{2}+I_{3}-I_{4}+I_{5}
$$

of the set (16.3) becomes, in terms of components relative to any coördinate plane,

$$
2\left|\begin{array}{cc}
i^{\prime} w & i^{\prime} z \\
k^{\prime} w & k^{\prime} z
\end{array}\right|=\left|\begin{array}{cc}
i w & i z \\
k w & k z
\end{array}\right|+\left|\begin{array}{cc}
i w & i z \\
l w & l z
\end{array}\right|-\left|\begin{array}{cc}
j w & j z \\
k w & k z
\end{array}\right|+\left|\begin{array}{cc}
l w & l z \\
j w & j z
\end{array}\right| .
$$

When the determinants on the right are collected this becomes

$$
2\left|\begin{array}{cc}
i^{\prime} w & i^{\prime} z \\
k^{\prime} w & k^{\prime} z
\end{array}\right|=\left|\begin{array}{cc}
(i-j) w & (i-j) z \\
(k+l) w & (k+l) z
\end{array}\right| .
$$

This equation states in components that the elementary six-vector $2 I_{2}{ }^{\prime}$ is the same as one whose plane contains $i-j$ and $k+l$. Therefore $i^{\prime}$ and $k^{\prime}$ belong to the plane of $i-j$ and $k+l$, and for some four numbers $\alpha, \beta, \gamma, \delta$ we have

$$
i^{\prime}=\alpha(i-j)+\beta(k+l), k^{\prime}=\gamma(i-j)+\delta(k+l) .
$$

But since $I_{1}^{\prime}=I_{1}, i^{\prime}$ and $j^{\prime}$ lie in the plane of $i$ and $j$. Likewise $I_{6}^{\prime}=I_{6}$ so $k^{\prime}$ and $l^{\prime}$ lie in the plane of $k$ and $l$. Hence for some four numbers $\lambda, \mu, \epsilon, \eta$ we have

$$
i^{\prime}=\lambda i+\mu j, \quad k^{\prime}=\epsilon k+\eta l,
$$

with similar expressions for $j^{\prime}$ and $l^{\prime}$. .

Since the vectors involved are unit vectors the last four equations determine the values of the eight numbers except for ambiguous signs, and these affect only the senses of $i^{\prime}$ and $j^{\prime}$. Similarly the equation

$$
2 I_{6}^{\prime}=I_{2}-I_{3}+I_{4}+I_{5}
$$

gives $j^{\prime}$ and $l^{\prime}$ in terms of the old unit vectors, and the remaining equations in (16.3) give nothing new. The relations thus found are

$$
\begin{aligned}
& i^{\prime}=(i-j) / 2^{1 / 2}, \quad j^{\prime}=(i+j) / 2^{1 / 2}, \\
& k^{\prime}=(k+l) / 2^{1 / 2}, \quad l^{\prime}=(k-l) / 2^{1 / 2} .
\end{aligned}
$$

Hence $i, j, k, l$ can be made to coincide with $i^{\prime}, j^{\prime}, k^{\prime}, l^{\prime}$ by rotating $i$ and $j$ through an angle $\pi / 4$ in the $i, j$ plane and $k$ and $l$ through $-\pi / 4$ in the $k, l$ plane. If $\pi / 4$ is used in both rotations the skeletons will still be made to coincide.

We can always arrange our passage from one set of intrinsic coördinate six-vectors to another so as to have two relations of the type $I_{1}^{\prime}=I_{1}, I_{6}^{\prime}=I_{6}$ 
among the old and new vectors. Hence we can generalize the above method to one for passing from any skeleton to another by rotations, and we can count just six distinct skeletons to be found in this manner.

THEOREM 9. Given one skeleton of $G(x, y ; u, v)$ we can rotate its axes through $\pi / 4$ in any one of its six planes and through the same angle in the absolutely perpendicular plane to obtain a new skeleton. We may proceed in the same way with any new skeleton to get another, but there are just six distinct skeletons to be found in this way.

The relations between the coefficients $\alpha_{i}^{\prime}, \beta_{i}^{\prime}$ for the new skeleton and $\alpha_{i}$, $\beta_{i}$ for the old can be found by observing from (14.7) that these coefficients are the values which the tensor

$$
F(d, m)=G(d, m)-d m R / 12
$$

assumes when the intrinsic coördinate six-vectors are its arguments. When components are referred to the skeleton defined by (16.2) we have

(16.4) $F(d, m)=\alpha_{\rho}^{\prime}\left(d_{\rho}^{\prime} m_{\rho}^{\prime}+d_{\eta_{-\rho}}^{\prime} m_{-\rho}^{\prime}\right)+\beta_{\rho}^{\prime}\left(d_{\rho}^{\prime} m_{\eta_{-\rho}}^{\prime}+d_{7_{-}}^{\prime} m_{\rho}^{\prime}\right)(\rho=1,2,3)$.

Now by referring to (16.3) we find that

$$
\begin{aligned}
\alpha_{1}^{\prime} & =F\left(I_{1}^{\prime}, I_{1}^{\prime}\right)=F\left(I_{1}, I_{1}\right)=\alpha_{1}, \\
\alpha_{2}^{\prime}=F\left(I_{2}^{\prime}, I_{2}^{\prime}\right) & =\left[F\left(I_{2}, I_{2}\right)+F\left(I_{3}, I_{3}\right)+F\left(I_{2}, I_{5}\right)-F\left(I_{3}, I_{4}\right)\right] / 2 \\
& =\left(\alpha_{2}+\alpha_{3}+\beta_{2}-\beta_{3}\right) / 2 .
\end{aligned}
$$

In the same way we find the relations

$$
\begin{aligned}
& \alpha_{3}^{\prime}=\left(\alpha_{2}+\alpha_{3}-\beta_{2}+\beta_{3}\right) / 2, \beta_{1}^{\prime}=\beta_{1}, \\
& \beta_{2}^{\prime}=\left(\beta_{2}+\beta_{3}+\alpha_{2}-\alpha_{3}\right) / 2, \beta_{3}^{\prime}=\left(\beta_{2}+\beta_{3}-\alpha_{2}+\alpha_{3}\right) / 2 .
\end{aligned}
$$

From the relations between the coördinate six-vectors of any two skeletons the relations between the two sets of coefficients can be written by the above method.

17. Cases of multiple roots. We have shown that the tensor of the first type has at least six skeletons, and just six if the roots of the characteristic equation (11.1) are distinct. Let us determine the number of skeletons for the various cases of multiple roots. The number of independent scalars needed in each case to complete the description of the tensor is the number of these roots which are independent after their multiplicity and the relation (13.9) have been considered.

It was shown that there is at least one set of mutually perpendicular invariable directions for $G(A)$ for which the vectors $A^{\prime}, B^{\prime}, C^{\prime}$ along three of them are self-dual and the vectors $A^{\prime \prime}, B^{\prime \prime}, C^{\prime \prime}$ along the other three are 
anti-self-dual. Three of the roots, $\alpha^{\prime}, \beta^{\prime}, \gamma^{\prime}$, of (11.1) are the multipliers of the vectors of the first group and the other three, $\alpha^{\prime \prime}, \beta^{\prime \prime}, \gamma^{\prime \prime}$, are the multipliers for the second group.

If multiple roots appear only to the extent that two of the same group are equal, say $\alpha^{\prime}=\beta^{\prime}$, then it follows from (12.1) that any six-vector of the plane of $A^{\prime}$ and $B^{\prime}$ is of invariable direction. Since every vector of this plane is a three-vector of the same type as $A^{\prime}$ and $B^{\prime}$, any pair of perpendicular vectors of square 2 in this plane helps to determine six skeletons for the tensor just as $A^{\prime}$ and $B^{\prime}$ did. There is a one-parameter family or simple infinity of such pairs in this plane, so there is a simple infinity of skeletons for the tensor in this case.

The number of skeletons is not increased due to the equality of two multipliers of opposite groups. For if $\alpha^{\prime}=\alpha^{\prime \prime}$ then every vector in the plane of $A^{\prime}$ and $A^{\prime \prime}$ is of invariable direction with $\alpha^{\prime}$ as multiplier, but $A^{\prime}$ and $A^{\prime \prime}$ determine the only two directions in this plane whose vectors are three-vectors. Hence $A^{\prime}$ and $B^{\prime}$ are the only vectors of this plane which can be used in determining skeletons. Multiplicity of this type then causes only a reduction in the number of scalars needed to describe the tensor.

By the above method it is easy to determine the number of skeletons corresponding to each kind of multiplicity of the roots. The condition (13.9) helps to reduce the number of cases in which more than six skeletons exist to five. The results are given in tabular form below.

$\begin{array}{lll}\text { 1. } & \alpha^{\prime}=\beta^{\prime} & \infty \text { skeletons } \\ \text { 2. } & \alpha^{\prime}=\beta^{\prime}, \alpha^{\prime \prime}=\beta^{\prime \prime} & \infty^{2} \text { “ } \\ \text { 3. } & \alpha^{\prime}=\beta^{\prime} ; \alpha^{\prime \prime}=\beta^{\prime \prime}=\gamma^{\prime \prime} & \infty^{4} \text { “ } \\ \text { 4. } & \alpha^{\prime}=\beta^{\prime}=\gamma^{\prime} & \infty^{3} \text { “ } \\ \text { 5. } & \alpha^{\prime}=\beta^{\prime}=\gamma^{\prime}=\alpha^{\prime \prime}=\beta^{\prime \prime}=\gamma^{\prime \prime} & \infty^{6} \text { “ }\end{array}$

In this table $\alpha^{\prime}, \beta^{\prime}, \gamma^{\prime}$ denote the roots of either group and $\alpha^{\prime \prime}, \beta^{\prime \prime}, \gamma^{\prime \prime}$ those of the opposite. This is not just a convention to shorten the table, for three-vectors of the self-dual type can be made anti-self-dual by reversing the sense of some of the coördinate axes. Three-vectors of opposite types remain of opposite types for any coördinate transformation.

\section{The TENSOR OF THE SECOND TYPE}

18. Four-vector geometry of this tensor. A set of four mutually perpendicular directions* which are intrinsically related to the second part of the Riemann tensor become evident at once when the second part, $E(a, b)$ or

* These directions are due to Ricci, loc. cit. 
$E(x, y ; u, v)$, is expressed in terms of the first and second contracted Riemann tensor, $R(w, z)$ and $R$. This expression,

$$
\begin{aligned}
E(x, y ; u, v)=\left|\begin{array}{cc}
R(x, u) & R(x, v) \\
y u & y v
\end{array}\right| / 2+ & \left|\begin{array}{cc}
x u & x v \\
R(y, u) & R(y, v)
\end{array}\right| / 2 \\
& -\left|\begin{array}{cc}
x u & x v \\
y u & y v
\end{array}\right| R / 4,
\end{aligned}
$$

has been written in components and verified by Einstein.*

According to (3.5) and the definition (3.10) of the first contracted Riemann tensor, this tensor is symmetric, $R(w, z)=R(z, w)$. It is therefore determined by four mutually perpendicular intrinsic directions and four numbers (cf. $\$ 10)$. Let coördinate vectors $i, j, k, l$ be chosen along these directions. Then, according to (10.6),

$$
R(w, z)=\omega_{\alpha} w_{\alpha} z_{\alpha} \quad(\alpha=1,2,3,4),
$$

where $\omega_{1}, \omega_{2}, \omega_{3}, \omega_{4}$ are the characteristic numbers corresponding to the directions of $i, j, k, l$, respectively, and $w_{p}, z_{p}$ are the components of $w, z$ relative to this intrinsic coördinate system. The second contracted Riemann tensor can be written

$$
R=\omega_{1}+\omega_{2}+\omega_{3}+\omega_{4} .
$$

Now let the first contracted Riemann tensor in the determinants of (18.1) be expressed in the form (18.2). The symbol $\omega_{\alpha}$ can be removed as a factor from the first two determinants, and when the third is broken into equal parts (18.1) becomes

$$
\begin{aligned}
E(x, y ; u, v)= & \left(\left|\begin{array}{cc}
x_{\alpha} u_{\alpha} & x_{\alpha} v_{\alpha} \\
y u & y v
\end{array}\right|-\left|\begin{array}{cc}
y_{\alpha} u_{\alpha} & y_{\alpha} v_{\alpha} \\
x u & x v
\end{array}\right|\right) \omega_{\alpha} / 2 \\
& -\left(\left|\begin{array}{cc}
x_{\alpha} u_{\alpha} & x_{\alpha} v_{\alpha} \\
y u & y v
\end{array}\right|-\left|\begin{array}{cc}
y_{\alpha} u_{\alpha} & y_{\alpha} v_{\alpha} \\
x u & x v
\end{array}\right|\right) R / 8 \quad(\alpha=1,2,3,4) .
\end{aligned}
$$

Here the value of the entire right-hand member for $\alpha=1$ is to be added to the value for $\alpha=2$, etc. For any $\alpha$ the part in parentheses can be factored out, and one scalar and one vector factor can be removed from each determinant:

$$
E(x, y ; u, v)=\left(\omega_{\alpha}-R / 4\right)\left(x_{\alpha} y\left|\begin{array}{ll}
u_{\alpha} & v_{\alpha} \\
u & v
\end{array}\right|-y_{\alpha} x\left|\begin{array}{ll}
u_{\alpha} & v_{\alpha} \\
u & v
\end{array}\right|\right) / 2 .
$$

The determinants here are vectors. Let these be factored out and the coefficients simplified by writing

* Loc. cit. 


$$
\left(\omega_{p}-R / 4\right) / 2=\gamma_{p} \quad(p=1,2,3,4) .
$$

The expression for the tensor of the second type then becomes

$$
E(x, y ; u, v)=\gamma_{\alpha}\left|\begin{array}{cc}
x_{\alpha} & y_{\alpha} \\
x & y
\end{array}\right|\left|\begin{array}{cc}
u_{\alpha} & v_{\alpha} \\
u & v
\end{array}\right| \quad(\alpha=1,2,3,4),
$$

where, according to (18.3),

$$
\gamma_{1}+\gamma_{2}+\gamma_{3}+\gamma_{4}=0 .
$$

The determinants in (18.5) are scalarly multiplied, and the value of the entire right-hand member for $\alpha=1$ is to be added to the value for $\alpha=2$, etc. We can summarize our results as follows:

ThEOREM 10. The tensor $E(x, y ; u, v)$ is determined by a skeleton and four numbers, one number corresponding to each direction of the skeleton, but only three of these numbers are independent. The tensor can be expressed in the form (18.5) by referring the components of its vector arguments to the intrinsic coordinate system determined by the skeleton.

By referring it to the intrinsic coördinate system the number of independent components of $E(x, y ; u, v)$ is reduced from nine to three.

Special cases arise when the four characteristic numbers of $R(w, z)$ are not all distinct. If two of these numbers are equal, say $\omega_{1}=\omega_{2}$, then $R(w, z)$ has a principal plane. Any two perpendicular directions in this plane together with the two corresponding to $\omega_{3}$ and $\omega_{4}$ form a skeleton for $E(x, y ; u, v)$. In this case $E(x, y ; u, v)$ has a simple infinity of skeletons and only two of the four numbers $\gamma_{p}$ are independent.

If three of the characteristic numbers are equal, the tensor of the second type can be described by means of any one of a triple infinity of skeletons and one number; but if all four are equal it follows from (18.4) and (18.6) that the tensor vanishes. In case of two pairs of equal characteristic numbers the tensor can be described by any one of a double infinity of skeletons and one number.

19. Six-vector geometry of this tensor. Each set of unit coördinate fourvectors determines a set of unit coördinate six-vectors. Hence the tensor of the second type is determined by a six-vector skeleton and three independent numbers, and to express it in terms of these quantities it is only necessary to write (18.5) in terms of elementary six-vectors and their components relative to the intrinsic coördinate system.

Let $d$ and $m$ be the elementary six-vectors determined by the pairs of fourvectors $x, y$ and $u, v$, respectively; then 


$$
E(x, y ; u, v)=E(d, m)=E_{\rho \sigma} d_{\rho} m_{\sigma} \quad(\rho, \sigma=1,2, \cdots, 6),
$$

where

$$
E_{11}=E\left(I_{1}, I_{1}\right)=E(i, j ; i, j)=-E_{68}, \quad E_{12}=E(i, j ; i, k)=-E_{65},
$$

etc. By using the unit coördinate four-vector form of these components it is found from (18.5) that $E(d, m)$, when referred to its skeleton, has only three independent non-vanishing components:

$$
E_{11}=\gamma_{1}+\gamma_{2}, E_{22}=\gamma_{1}+\gamma_{3}, E_{33}=\gamma_{1}+\gamma_{4} .
$$

When these are used in (19.1) we find

$$
\begin{aligned}
E(d, m)=\left(\gamma_{1}+\right. & \left.\gamma_{2}\right)\left(d_{1} m_{1}-d_{6} m_{6}\right) \\
& +\left(\gamma_{1}+\gamma_{3}\right)\left(d_{2} m_{2}-d_{5} m_{5}\right)+\left(\gamma_{1}+\gamma_{4}\right)\left(d_{3} m_{3}-d_{4} m_{4}\right) .
\end{aligned}
$$

This is the form of the tensor of the second type when referred to its sixvector skeleton.

\section{Conclusion}

The Riemann tensor is determined by two intrinsic sets of orthogonal directions, or skeletons, and eight numbers. This tensor is the sum of three parts,

$$
R(x, y ; u, v)=H(x, y ; u, v)+F(x, y ; u, v)+E(x, y ; u, v) .
$$

The first of these is determined by just one number, the second contracted Riemann tensor. The second is determined by one of the skeletons and four numbers, and the third by the other skeleton and three numbers.

In the general case there is just one skeleton for the tensor $E(x, y ; u, v)$. But from one skeleton and four numbers which determine $F(x, y ; u, v)$ five other skeletons and sets of numbers, bearing the same intrinsic relation to this tensor as the first, can be obtained. The tensor $H(x, y ; u, v)$ is given by (15.2) independently of any coördinate system. $F(x, y ; u, v)$ is given by (15.3) when referred to the coördinate system determined by one of its skeletons, and $E(x, y ; u, v)$, referred to its skeleton, is given by (18.5).

In special cases the number of skeletons may form an $m$-parameter family with $m=1,2,3,4$ or 6 for $F(x, y ; u, v)$ and $m=1,2$ or 3 for $E(x, y ; u, v)$.

UNIVERSITY OF MICHIGAN,

ANn Arbor, Mich. 